\title{
Securing Communication via Transmission of Artificial Noise by Both Sides: Bipolar-Beamforming Optimization
}

\author{
Yongkai Zhou, ${ }^{1}$ Yan Zhu, ${ }^{1}$ Fangbiao Li, ${ }^{1}$ Zachary Imm, ${ }^{2}$ Xinxing ${ }^{1}{ }^{1},{ }^{1}$ and Zhi Xue \\ ${ }^{1}$ Department of Electronic Engineering, Shanghai Jiao Tong University, Shanghai 200240, China \\ ${ }^{2}$ Department of Electronic Engineering, Ohio State University, Columbus, OH 43210, USA \\ Correspondence should be addressed to Yongkai Zhou; ssmailzyk@sjtu.edu.cn
}

Received 30 August 2013; Accepted 8 November 2013

Academic Editor: Hao Shen

Copyright (c) 2013 Yongkai Zhou et al. This is an open access article distributed under the Creative Commons Attribution License, which permits unrestricted use, distribution, and reproduction in any medium, provided the original work is properly cited.

\begin{abstract}
The paper considers the secure transmission in a wireless environment in which both the transmitter (Alice) and the legitimate receiver (Bob) send artificial noise (AN) to interfere with the eavesdropper (Eve). Optimal design is analyzed in detail for this AN-by-both-side model to deal with Eve's stochastic channel condition and random spatial distribution. Bipolar-beamforming is first proposed to jointly design Alice and Bob's transmitting signals. By optimally assigning the transmitting antenna for Bob and allocating the power ratio between Alice's information and the AN signal, maximum secrecy capacity can be achieved. Simulation is done to illustrate the process of bipolar-beamforming optimization. Results show that the AN-by-both-side model has good secrecy performance on both average and extreme conditions as Eve approaches Alice or Bob.
\end{abstract}

\section{Introduction}

In wireless communication, a malicious intruder can easily overhear messages transmitted between two nodes. Traditional security problems involve three parties: transmitter (Alice), legitimate receiver (Bob), and a passive eavesdropper (Eve). Wyner [1] first studied the wiretap channel and introduced the notion of secrecy capacity. After that, Csiszár and Körner [2] extended this work to the nondegraded discrete memoryless broadcast channel. Their work showed that if Bob's channel is more capable than Eve's, perfect secrecy can be achieved. Secrecy capacity in MIMO (multiple-input and multiple-output) wiretap channel and fading channel is studied in $[3,4]$. Recently, the problem of stochastic system modeling has also been considered in [5-11].

The method of adopting artificial noise (AN) to increase secrecy capacity was first proposed by Goel and Negi $[12,13]$. In their model, Alice uses multiple transmitting antennas or cooperates with the helper nodes to generate the AN, shown in Figure 1(a). This degrades Eve's channel, without affecting Bob, by projecting the AN into the null space of Bob's channel. Authors in [14-17] adopted an iterative waterfilling algorithm to allocate the power ratio between $\mathrm{AN}$ and the information signal, thus solving the optimal design problem of this model.

$\mathrm{Li}$ et al. proposed another AN based approach [18], in which AN is generated by Bob, shown in Figure 1(b). Such a kind of AN impairs Eve's channel but can be cancelled by Bob himself by applying the self-interference cancellation techniques [19-21]. In their model, Bob adopts one antenna for receiving information and another for transmitting AN. Outage secrecy capacity region (OSR) is analyzed for such a configuration. Li et al. also mentioned a model in the discussion part of their paper in which both Alice and Bob send AN. As shown in Figure 1(c), this is a combination of the above two models. However, no detailed optimization analysis is available yet for this AN-by-both-side model.

In this paper, we will generalize the above AN-by-bothside model from Alice equipped with 2 antennas, Bob with 2 antennas, and Eve with 1 antenna to a model that all of them are equipped with multiple antennas. Optimal design will be studied for such a configuration to best utilize Alice and Bob's antenna and power resources. In order to achieve optimal secrecy performance, many design-specific issues need to be addressed to deal with a stochastic Eve with random channel condition and spatial distribution. These 


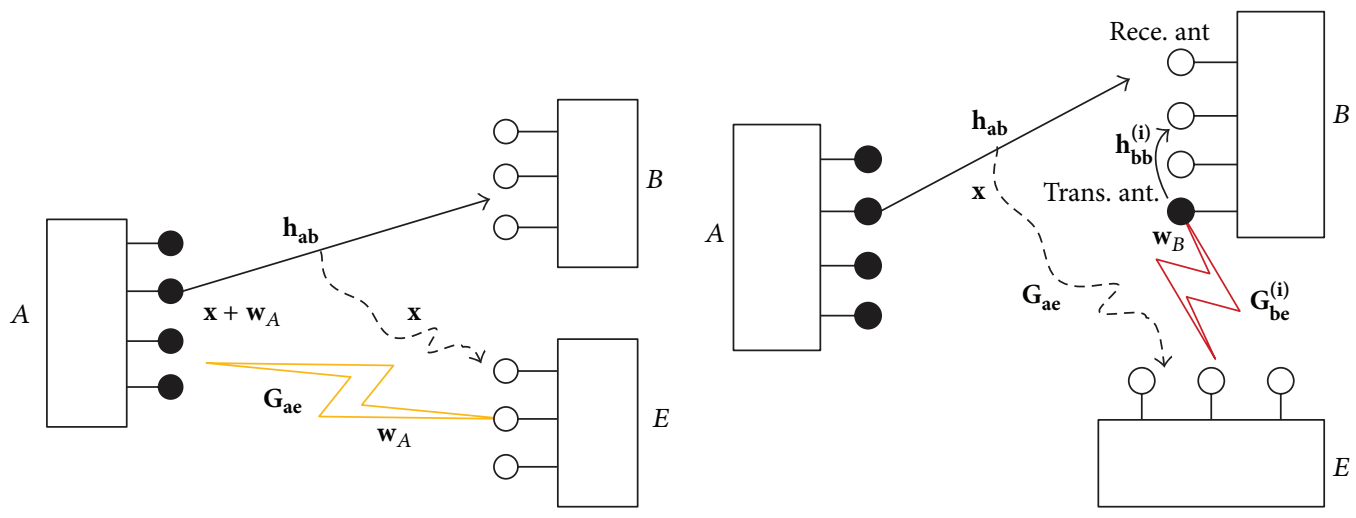

(a) AN sent by Alice

(b) AN sent by Bob

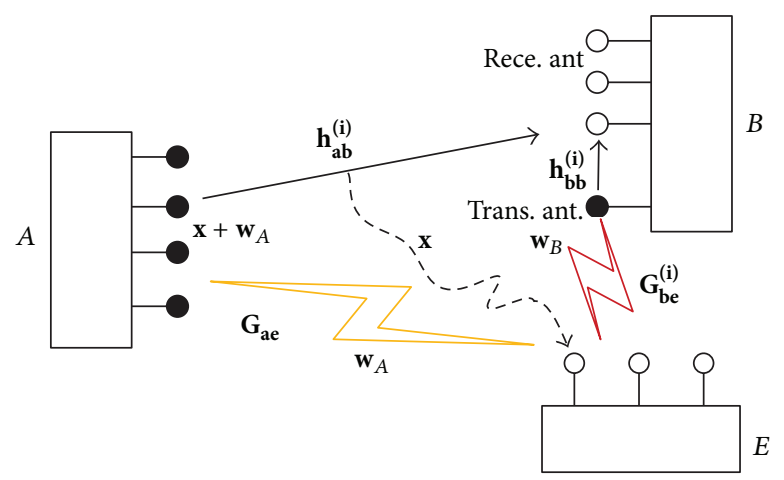

(c) AN sent by both sides

FIGURE 1: Illustration to compare 3 different kinds of AN models.

include the assignment of Bob's antennas, the optimal power allocation ratio for Alice, and the beamforming design. In this work, we will propose an efficient scheme, called "bipolar-beamforming optimization," to design Alice and Bob's transmitting signals and allocate their antenna and power resources so that maximum secrecy capacity can be achieved.

The paper is organized as follows. The system model will be described in Section 2. Sections 3-5 will then focus on the efficient computation of secrecy capacity and optimal parameter design for the proposed model. Simulation results will be presented in Section 6, and the conclusion will be given in Section 7.

Throughout this paper, the following notations will be used: bold symbols (e.g., $\mathbf{G}$ and $\mathbf{g}$ ) in capital letter and small letter denote matrices and vectors, respectively. $\mathbb{C}^{N}$ is the $N$ dimension vector space over the complex field. $(\cdot)^{\dagger}$ denotes the conjugate transpose and $(x)^{+}=\max \{0, x\} .|\cdot|$ is the determinant of a square matrix while $\|\cdot\|$ is the norm of a vector. $E_{\mathbf{G}}(\cdot)$ stands for the expectation over the random variate $\mathbf{G}$.

\section{System Model}

Suppose Alice is equipped with $N_{A}\left(N_{A} \geq 2\right)$ antennas. Bob has $N_{B}\left(N_{B} \geq 2\right)$ antennas. He will choose the $i$ th $(1 \leq i \leq$ $N_{B}$ ) antenna as receiving antenna, and the remaining ones as
AN-emitting antennas. Eve will have $N_{E}\left(N_{E} \geq 1\right)$ antennas for eavesdropping on the transmission signal between Alice and Bob. It is assumed that the channel between Alice and Bob is known to all parties, but they do not hold channel state information (CSI) of Eve.

As shown in Figure 1(c), when both Alice and Bob send $\mathrm{AN}$, the signal received by Bob can be expressed as

$$
y_{b}=\mathbf{h}_{\mathbf{a b}}^{(\mathbf{i})} \cdot \mathbf{x}+\mathbf{h}_{\mathbf{b b}}^{(\mathbf{i})} \cdot \mathbf{n}+n_{b},
$$

where $\mathbf{h}_{\mathbf{a b}}^{(\mathbf{i})}$ denotes the channel between Alice and the $i$ th receiving antenna of Bob, which is a $1 \times N_{A}$ vector. $\mathbf{h}_{\mathbf{b b}}^{(\mathbf{i})}$ is the channel between the receiving and the transmitting antennas of Bob, with size $1 \times\left(N_{B}-1\right) . n_{b}$ is the Gaussian noise at Bob, with noise power $\sigma_{n_{b}}^{2}$. The signal transmitted by Alice is $\mathbf{x}$, while $\mathbf{n}$ stands for the artificial noise emitted by Bob.

Similarly, the signal received by a stochastic Eve is given by

$$
\mathbf{y}_{\mathrm{e}}=\mathrm{G}_{\mathrm{ae}} \cdot \mathbf{x}+\mathrm{G}_{\mathrm{be}}^{(\mathbf{i})} \cdot \mathbf{n}+\mathbf{n}_{\mathrm{e}},
$$

where $\mathbf{n}_{\mathbf{e}}$ is the Gaussian noise at Eve. $\mathbf{G}_{\mathbf{a}}$ is the random channel between Alice's transmitting antennas and Eve. $G_{\text {be }}^{(i)}$ is the random channel between Bob's transmitting antenna set and Eve. The elements of $\mathbf{G}_{\mathbf{a e}}$ and $\mathbf{G}_{\mathbf{b e}}^{(\mathbf{i})}$ are modeled as i.i.d. zero-mean circularly symmetric complex Gaussian (ZMCSCG) random variables. 
The total transmission power of Alice and Bob are $P_{A}$ and $P_{B}$, respectively. For $P_{A}$, a proportion of $\phi(0 \leq \phi \leq 1)$ is allocated to the information signal, with others left for AN. Therefore, the power used for transmitting information and emitting $\mathrm{AN}$ is

$$
\begin{gathered}
P_{\text {info }}=\phi \cdot P_{A}, \\
P_{\mathrm{AN}}=(1-\phi) \cdot P_{A} .
\end{gathered}
$$

\section{Beamforming and Secrecy Capacity}

In this section, the beamforming of Alice and Bob's transmission signal is first studied. Secrecy capacity of the proposed AN-by-both-side model can therefore be obtained based on the signals received by Bob and Eve.

3.1. Bipolar Beamforming. For Bob, since the location and channel condition of Eve is unknown to him, the beamforming of $\mathbf{n}$ is just to generate random Gaussian noise. Therefore, n becomes an $\left(N_{B}-1\right) \times 1$ complex random Gaussian vector with covariance matrix $E\left(\mathbf{n n}^{\dagger}\right)=P_{B} /\left(N_{B}-1\right) \cdot \mathbf{I}_{N_{B}-1}$.

For Alice, the transmitted signal $\mathbf{x}$ can be decomposed into the information part, $\mathbf{s}$, and the AN part, $\mathbf{w}$ as follows:

$$
\mathbf{x}=\mathbf{s}+\mathbf{w}=\mathbf{w}_{1} \cdot u+\mathbf{W}_{2} \cdot \mathbf{v}
$$

According to the beamforming scheme by [13], ( $\left.\mathbf{w}_{1}, \mathbf{W}_{2}\right)$ forms an orthogonal basis of $\mathbb{C}^{N_{A}}$, and $\mathbf{w}_{\mathbf{1}}=\mathbf{h}_{\mathrm{ab}}^{(\mathbf{i}) \dagger} /\left\|\mathbf{h}_{\mathrm{ab}}^{(\mathbf{i})}\right\| . \mathbf{W}_{\mathbf{2}}$ spans the null space of $\mathbf{h}_{\mathrm{ab}}^{(\mathbf{i}) \dagger} \cdot u$ stands for the information symbol with power $E\left(u u^{\dagger}\right)=\sigma_{u}^{2}=P_{\text {info }}$; and $\mathbf{v}$ is a complex Gaussian vector with zero mean and covariance matrix $E\left(\mathbf{v v}^{\dagger}\right)=P_{\mathrm{AN}} /\left(N_{A}-1\right) \cdot \mathbf{I}_{N_{A}-1}$. Thus, (1) and (2) can be further expanded as

$$
\begin{aligned}
y_{b}(k) & =\mathbf{h}_{\mathbf{a b}}^{(\mathbf{i})} \cdot\left(\mathbf{w}_{\mathbf{1}} \cdot u+\mathbf{W}_{\mathbf{2}} \cdot \mathbf{v}\right)+\mathbf{h}_{\mathbf{b b}}^{(\mathbf{i})} \cdot \mathbf{n}+n_{b} \\
& =\left\|\mathbf{h}_{\mathbf{a b}}^{(\mathbf{i})}\right\| \cdot u+\mathbf{h}_{\mathbf{b b}}^{(\mathbf{i})} \cdot \mathbf{n}+n_{b}, \\
\mathbf{y}_{\mathbf{e}}(k) & =\mathbf{G}_{\mathbf{a e}} \cdot\left(\mathbf{w}_{\mathbf{1}} \cdot u+\mathbf{W}_{\mathbf{2}} \cdot \mathbf{v}\right)+\mathbf{G}_{\mathbf{b e}}^{(\mathbf{i})} \cdot \mathbf{n}+\mathbf{n}_{\mathbf{e}} \\
& =\mathbf{g} \cdot u+\mathbf{G} \cdot \mathbf{v}+\mathbf{G}_{\mathbf{b e}}^{(\mathbf{i})} \cdot \mathbf{n}+\mathbf{n}_{\mathbf{e}},
\end{aligned}
$$

where we denote $\mathbf{g}=\mathbf{G}_{\mathbf{a e}} \cdot \mathbf{w}_{\mathbf{1}}$ and $\mathbf{G}=\mathbf{G}_{\mathbf{a e}} \cdot \mathbf{W}_{\mathbf{2}}$ for simplicity. Note that due to the orthonormality of $\left(\mathbf{w}_{1}, \mathbf{W}_{2}\right), \mathbf{g}$ and $\mathbf{G}$ also have i.i.d. ZMCSCG entries as $\mathbf{G}_{\mathbf{a e}}$.

3.2. Secrecy Capacity. Based on the work of $[4,13]$, the lower bound of the secrecy capacity can be calculated in terms of (5), as follows:

$$
\begin{aligned}
& C s=\left(C s_{1}-\overline{C s_{2}}\right)^{+}, \\
& C s_{1}=\log _{2}\left(1+\mathbf{h}_{\mathbf{a b}}^{(\mathbf{i})} \cdot E\left(u u^{\dagger}\right) \cdot \frac{\mathbf{h}_{\mathbf{a b}}^{(\mathbf{i}) \dagger}}{\sigma_{n_{b}}^{2}}\right) \\
& =\log _{2}\left(1+\mathbf{h}_{\mathrm{ab}}^{(\mathbf{i})} \cdot P_{\mathrm{info}} \cdot \frac{\mathbf{h}_{\mathrm{ab}}^{(\mathbf{i}) \dagger}}{\sigma_{n_{b}}^{2}}\right) \\
& =\log _{2}\left(1+\phi \cdot P_{A} \cdot \frac{\left\|\mathbf{h}_{\mathbf{a b}}^{(\mathbf{i})}\right\|^{2}}{\sigma_{n_{b}}^{2}}\right) \\
& \overline{C s_{2}}=E_{\mathbf{G}_{\mathbf{b e}}^{(\mathbf{i})} \mathbf{g}, \mathbf{G}} \log _{2}\left|\mathbf{I}_{N_{E}}+\frac{\mathbf{g} \cdot E\left(u u^{\dagger}\right) \cdot \mathbf{g}^{\dagger}}{\mathbf{G} E\left(\mathbf{v v}^{\dagger}\right) \mathbf{G}^{\dagger}+\mathbf{G}_{\mathbf{b e}}^{(\mathbf{i})} E\left(\mathbf{n n}^{\dagger}\right) \mathbf{G}_{\mathbf{b e}}^{(\mathbf{i}) \dagger}}\right| \\
& =E_{\mathbf{G}_{\mathbf{b e}}^{(\mathbf{i})} \mathbf{g}, \mathbf{G}} \log _{2}\left|\mathbf{I}_{N_{E}}+\frac{P_{\text {info }} \cdot \mathbf{g g}^{\dagger}}{\left(P_{A N} /\left(N_{A}-1\right)\right) \mathbf{G G}^{\dagger}+\left(P_{B} /\left(N_{B}-1\right)\right) \mathbf{G}_{\mathbf{b e}}^{(\mathbf{i})} \mathbf{G}_{\mathbf{b e}}^{(\mathbf{i}) \dagger}}\right| \\
& =E_{\mathbf{G}_{\mathbf{b e}}^{(\mathbf{i})}, \mathbf{g}, \mathbf{G}} \log _{2}\left|\mathbf{I}_{N_{E}}+\frac{\phi \cdot P_{A} \cdot \mathbf{g g}^{\dagger}}{\left((1-\phi) P_{A} /\left(N_{A}-1\right)\right) \mathbf{G G}^{\dagger}+\left(P_{B} /\left(N_{B}-1\right)\right) \mathbf{G}_{\mathbf{b e}}^{(\mathbf{i})} \mathbf{G}_{\mathbf{b e}}^{(\mathbf{i}) \dagger}}\right| \\
& =E_{\mathbf{G}_{\mathbf{b e}}^{(\mathbf{i})} \mathbf{g}, \mathbf{G}} \log _{2}\left|\mathbf{I}_{N_{E}}+\phi \cdot \mathbf{g}\left[\mathbf{g}^{\dagger} \cdot\left(\frac{1-\phi}{N_{A}-1} \mathbf{G G}^{\dagger}+\frac{P_{B}}{P_{A} \cdot\left(N_{B}-1\right)} \mathbf{G}_{\mathbf{b e}}^{(\mathbf{i})} \mathbf{G}_{\mathbf{b e}}^{(\mathbf{i}) \dagger}\right)^{-1}\right]\right| \\
& \stackrel{(a)}{=} E_{\mathbf{G}_{\mathbf{b e}}^{(\mathbf{i})}, \mathbf{g}, \mathbf{G}} \log _{2}\left|1+\phi \cdot \mathbf{g}^{\dagger}\left(\alpha \mathbf{G G}^{\dagger}+\beta \mathbf{G}_{\mathbf{b e}}^{(\mathbf{i})} \mathbf{G}_{\mathbf{b e}}^{(\mathbf{i}) \dagger}\right)^{-1} \mathbf{g}\right|,
\end{aligned}
$$

where $\alpha=(1-\phi) /\left(N_{A}-1\right)$ and $\beta=P_{B} /\left(P_{A} \cdot\left(N_{B}-1\right)\right)$. (a) holds due to the determinant identity $|\mathbf{I}+\mathbf{A B}|=|\mathbf{I}+\mathbf{B A}|$.
Note that in (7), there is no $\mathbf{h}_{\mathbf{b b}}^{(\mathbf{i})} \cdot \mathbf{n}$ item in the calculation of $C s_{1}$ because it is possible for Bob to cancel the AN exerted 
on himself by adopting full duplex wireless communication techniques [20], and the problem of self-interference cancellation has also been discussed in depth in $[18,21]$.

In (8), the worst case situation, $\mathbf{n}_{\mathbf{e}} \rightarrow \mathbf{0}$, is considered. Therefore, $\overline{C s_{2}}$ is the upper bound of the information tapped by Eve; and $C s$ in (6) is the minimum guaranteed secrecy capacity that can be ensured in this scenario.

\section{Optimization for Secrecy Capacity}

Through the secrecy capacity (Cs) analyzed in (6)-(8), the optimization problem can be formalized as

$$
\begin{array}{ll}
\max _{\phi, i} & C s(\phi, i) \\
\text { s.t. } & \phi \in(0,1), 1 \leq i \leq N_{B} .
\end{array}
$$

In order to maximize this secrecy capacity, the following 2 key parameters need to be optimized:

(1) $i$, with the $i$ th antenna selected as Bob's receiving antenna, and the others left as AN transmitting antennas;

(2) $\phi$, the power allocation ratio between Alice's information signal and AN.

As it can be seen from (6)-(8), it is hard to analyze Cs due to the existence of 3 random matrices in $\overline{C s_{2}}: \mathbf{g}, \mathbf{G}$ and $\mathbf{G}_{\mathbf{b e}}^{(\mathbf{i})}$. In the following part, we will exploit the random characteristics of Eve to optimize the above 2 parameters.

4.1. Antenna Assignment for Bob. We first exam $\overline{C s_{2}}$. In (8), the item related to $i$ is $\beta \mathbf{G}_{\mathbf{b e}}^{(\mathbf{i})} \mathbf{G}_{\mathbf{b e}}^{(\mathbf{i}) \dagger}$. Because the channel between Bob and Eve is unknown to Bob and considered to be randomized, any combination of Bob's AN transmitting antennas will result, on average, in the same effect. Hence, $\overline{C s_{2}}$ is irrelevant to the selection of antenna $i$.

On the other hand, for (7), it is simple to check that $C s_{1}$ is monotonically increasing with $\left\|\mathbf{h}_{\mathbf{a b}}^{(\mathbf{i})}\right\|$. Therefore, this item needs to be maximized.

Finally; the assignment of Bob's antenna resources has a relatively simple solution, that is,

$$
i=\arg \max _{i}\left\{\left\|\mathbf{h}_{\mathbf{a b}}^{(\mathbf{i})}\right\|\right\} .
$$

4.2. Power Allocation Ratio for Alice. The form of Cs in (6)(8) is hard to be computed due to the existence of 3 random matrices in $\overline{C s_{2}}: \mathbf{g}, \mathbf{G}$ and $\mathbf{G}_{\mathbf{b e}}^{(\mathbf{i})}$. In fact, they are related to Eve's stochastic channel condition and spatial distribution. In the following section, we will first derive and explore an efficient method to compute the secrecy capacity based on Eve's random characteristics. The optimal power allocation ratio, $\phi^{*}$, can be obtained thereafter.

4.2.1. Eve's Stochastic Channel Condition. As it can be seen in (8), $\overline{C s_{2}}$ is the expectation over the channel condition of Eve; that is, $\mathbf{G}_{\mathrm{ae}}$ and $\mathbf{G}_{\mathrm{be}}$. When taking into account the pathloss effect, $\mathbf{G}_{\mathrm{ae}}$ can be expanded as the product of a path-loss component, $\sigma_{a e}$, and a Gaussian fading component, $\widetilde{\mathbf{G}_{\mathbf{a e}}}$ as follows:

$$
\mathbf{G}_{\mathbf{a e}}=\sigma_{a e} \cdot \widetilde{\mathbf{G}_{\mathrm{ae}}}=\left(\lambda_{0} \cdot r_{a e}^{-\kappa}\right)^{1 / 2} \cdot \widetilde{\mathbf{G}_{\mathrm{ae}}} .
$$

In (11), $\lambda_{0}$ is a coefficient and $r_{a e}$ is the distance between Alice and Eve. $\kappa$ stands for the pass-loss exponent. The Gaussian fading component, $\widetilde{\mathbf{G}_{\mathrm{ae}}}$, is a unit random Gaussian matrix, and the elements of $\widetilde{\mathbf{G}_{\mathrm{ae}}}$ are normalized to be i.i.d. zero-mean unit-covariance circularly symmetric complex Gaussian random variables.

This is similar for $\mathbf{G}_{\mathbf{b e}}^{(\mathbf{i})}, \mathbf{g}$, and $\mathbf{G}$; that is,

$$
\begin{aligned}
\mathbf{G}_{\mathbf{b e}}^{(\mathbf{i})} & =\left(\lambda_{0} \cdot r_{b e}^{-\kappa / 2}\right) \cdot \widetilde{\mathbf{G}_{\mathbf{b e}}^{(\mathbf{i})}}, \\
\mathbf{G} & =\left(\lambda_{0} \cdot r_{a e}^{-\kappa / 2}\right) \cdot \widetilde{\mathbf{G}}, \\
\mathbf{g} & =\left(\lambda_{0} \cdot r_{a e}^{-\kappa / 2}\right) \cdot \widetilde{\mathbf{g}},
\end{aligned}
$$

where $\widetilde{\mathbf{G}_{\text {be }}^{(\mathbf{i})}}$, $\widetilde{\mathbf{g}}$, and $\widetilde{\mathbf{G}}$ are all normalized random Gaussian matrices, the same as $\widetilde{\mathbf{G}_{\mathrm{ae}}}$.

Therefore, by substituting (11)-(12), (8) can be further derived as (13) consider the following:

$$
\begin{aligned}
& \overline{C s_{2}}
\end{aligned}
$$

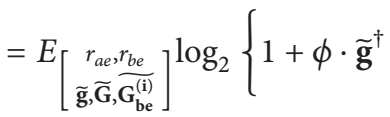

$$
\begin{aligned}
& \left.\cdot\left(\alpha \widetilde{\mathbf{G}} \widetilde{\mathbf{G}}^{\dagger}+\beta\left(\frac{r_{a e}}{r_{b e}}\right)^{\kappa} \widetilde{\mathbf{G}_{\mathbf{b e}}^{(\mathbf{i})}} \widetilde{\mathbf{G}_{\mathbf{b e}}^{(\mathbf{i})}}\right)^{\dagger} \widetilde{\mathbf{g}}\right\} \\
& =E_{\left[\begin{array}{c}
r_{a e}, r_{b e} \\
\widetilde{\mathbf{g}},{ }_{\mathbf{g}}, \underline{\mathbf{g}}_{m}
\end{array}\right]} \log _{2}\left\{+\sum_{m-1}^{N_{B}-1} \beta\left(\frac{r_{a e}}{r_{b e}}\right)^{\kappa} \widetilde{\mathbf{g}_{m}} \widetilde{\mathbf{g}}_{m}^{\dagger}\right)^{-1} 1+\phi \cdot \widetilde{\mathbf{g}}^{\dagger} \\
& \cdot\left(\sum_{l-1}^{N_{A}-1} \alpha \cdot \widetilde{\mathbf{g}}_{l} \widetilde{\mathbf{g}}_{l}^{\dagger}\right. \\
& \left.\left.+\sum_{m-1}^{N_{B}-1} \beta\left(\frac{r_{a e}}{r_{b e}}\right)^{\kappa} \widetilde{\mathbf{g}_{m}} \widetilde{\mathbf{g}}_{m}^{\dagger}\right)^{-1} \widetilde{\mathbf{g}}\right\} \\
& \stackrel{(b)}{=} E_{\left[\begin{array}{c}
r_{a e}, r_{b e} \\
\widetilde{\mathbf{g}}, \widetilde{\mathbf{g}_{k}}
\end{array}\right]} \log _{2}\left\{1+\phi \cdot \widetilde{\mathbf{g}}^{\dagger}\right. \\
& \left.\cdot\left(\sum_{k-1}^{N_{A}-1+N_{B}-1} \Gamma_{k} \widetilde{\mathbf{g}_{k}} \widetilde{\mathbf{g}}_{k}^{\dagger}\right)^{-1} \widetilde{\mathbf{g}}\right\} .
\end{aligned}
$$

In (13), the normalized Gaussian vector, $\widetilde{\mathbf{g}}_{l}\left(1 \leq l \leq N_{A}-\right.$ $1), \widetilde{\mathbf{g}_{m}}\left(1 \leq m \leq N_{B}-1\right)$, and $\widetilde{\mathbf{g}_{k}}\left(1 \leq k \leq N_{A}+N_{B}-2\right)$ are the column vectors of $\widetilde{\mathbf{G}}, \widetilde{\mathbf{G}_{\mathbf{b e}}^{(\mathbf{i})}}$, and $\left[\widetilde{\mathbf{G}}, \widetilde{\mathbf{G}_{\mathbf{b e}}^{(\mathbf{i})}}\right]$, with the following coefficients:

$$
\Gamma_{k}= \begin{cases}\Gamma_{1}=\alpha, & 1 \leq k \leq N_{A}-1, \\ \Gamma_{N_{A}}=\beta\left(\frac{r_{a e}}{r_{b e}}\right)^{\kappa}, & k \geq N_{A} .\end{cases}
$$


As a result, the item $X=\widetilde{\mathbf{g}}^{\dagger}\left(\sum_{k=1}^{N_{A}-N_{B}-2} \Gamma_{k} \cdot \widetilde{\mathbf{g}_{k}} \widetilde{\mathbf{g}}_{k}^{\dagger}\right)^{-1} \widetilde{\mathbf{g}}$ in (13) is equivalent to the signal-to-interference ratio of an $N_{E^{-}}$ branch MMSE diversity combiner having $\left(N_{A}-1\right)+\left(N_{B}-\right.$ 1) interfers with covariance matrix $\operatorname{diag}\left\{\Gamma_{1}, \ldots, \Gamma_{N_{A}-N_{B}-2}\right\}$. The complementary cumulative distribution function of $X$ is given in [22] (Equation (18) in that paper), as

$$
\begin{aligned}
R(x) & =\operatorname{Prob}\{X \geq x\}=\frac{1+\sum_{m=1}^{N_{E}-1} C_{m} x^{m}}{\prod_{k=1}^{N_{A}+N_{B}-2}\left(1+\Gamma_{k} x\right)} \\
& =\frac{\sum_{m=0}^{N_{E}-1} C_{m} x^{m}}{\prod_{k=1}^{N_{A}+N_{B}-2}\left(1+\Gamma_{k} x\right)} \\
& =\frac{\sum_{m=0}^{N_{E}-1} C_{m} x^{m}}{\left(1+\Gamma_{1} x\right)^{N_{A}-1}\left(1+\Gamma_{N_{A}} x\right)^{N_{B}-1}},
\end{aligned}
$$

where $C_{m}$ is the coefficient of $x^{m}$ in the polynomial, $\prod_{k=1}^{N_{A}+N_{B}-2}\left(1+\Gamma_{k} x\right)$. Hence, the probability density function of $X$ is $f_{X}(x)=\mathrm{d}(-R(x))$. We can rewrite the expectation in (13) into integral form as

$$
\begin{aligned}
\overline{C s_{2}}= & E_{r_{a e}, r_{b e}} \int_{0}^{\infty} \log _{2}(1+\phi x) \cdot \mathrm{d}(-R(x)) \\
\stackrel{(c)}{=} & E_{r_{a e}, r_{b e}} \frac{1}{\ln 2} \int_{0}^{\infty} \frac{\phi}{1+\phi x} R(x) d x \\
& +\left[\log _{2}(1+\phi x) R(x)\right]_{x=0}^{x=0} \\
= & E_{r_{a e}, r_{b e}} \frac{1}{\ln 2} \int_{0}^{\infty} \frac{\phi}{1+\phi x} R(x) d x+(0-0) \\
\stackrel{(d)}{=} & E_{r_{a e}, r_{b e}} \frac{1}{\ln 2} \sum_{m=0}^{N_{E}-1} \int_{0}^{\infty} \frac{C_{m} \cdot \phi \cdot x^{m} \cdot(1+\phi x)^{-1} d x}{\left(1+\Gamma_{1} x\right)^{N_{A}-1}\left(1+\Gamma_{N_{A}} x\right)^{N_{B}-1}}
\end{aligned}
$$

(c) is obtained using integration by parts. Note that for a given $r_{a e}$ and $r_{b e}$, the integrand in (16) corresponds to a rational function. The improper integral, therefore, can be easily computed by adopting partial fraction decomposition.

Also, note that the second term in $(c)$ equals 0 , because $\lim _{x \rightarrow 0} \log _{2}(1+\phi x) R(x)=0$. If the size constraint in Section 5.1 is fulfilled; that is, $\left(N_{A}-1\right)+\left(N_{B}-1\right) \geq N_{E}$,

$$
\lim _{x \rightarrow \infty} \log _{2}(1+\phi x) R(x) \sim \lim _{x \rightarrow \infty} \frac{\log _{2}(x)}{x^{N_{A}+N_{B}-N_{E}-1}}=0 .
$$

4.2.2. Spatial Distribution of Eve. In (16), $\overline{C s_{2}}$ is still the expectation over $r_{a e}$ and $r_{b e}$, which is in turn related to the spatial location of Eve. Therefore, some geometry distribution could be prespecified for this imaginary attacker. For example, in $[16,23]$, the location of Eve follows a Poisson point process.

In our simulation (see Section 6, Figure 4), however, a certain region of area within a radius of $2 \cdot r_{a b}$ is delimited around Alice and Bob. The probability density function of Eve's location can be expressed as

$$
p\left(E_{x, y}\right)= \begin{cases}\frac{1}{S(\mathscr{D})}, & E_{x, y} \in \mathscr{D}, \\ 0, & E_{x, y} \notin \mathscr{D},\end{cases}
$$

where $E_{x, y}$ is the coordinate of Eve's location and $\mathscr{D}=\left\{E_{x, y} \mid\right.$ $\left.r_{a e}, r_{b e}<2 \cdot r_{a b}\right\}$. We refer to $\mathscr{D}$ as the "Protected Zone," and $S(\mathscr{D})$ is the area of such a region. 25 points are sampled uniformly and symmetrically in this region to approximately compute the expectation in (16). Outside this region, Eve is considered to have little impact on the secure communication because the relatively high pass-loss and low-SNR makes it difficult to decode the information signal.

4.2.3. Searching for Optimal $\phi^{*}$. Through the above derivation, Cs can be computed efficiently given a specified $\phi$. Therefore, the optimal power allocation ratio, $\phi^{*}$, can be obtained by some searching techniques.

Analytically, it is hard to prove the concavity of $C s$ in the form of (16). However, it can be checked from Figure 2 that the resulted curve is concave. Although not strict, it can still be empirically treated as a concave problem in practical engineering. Therefore, one-dimension search technique such as binary search can be applied to find the optimal $\phi^{*}$.

\section{Discussion and Extension}

5.1. Constraints on Antenna Numbers. There are some size constraints on the number of antennas for Alice, Bob, and Eve in the proposed AN-by-both-side scenario.

First, as can be seen from (8), to avoid $\alpha \mathbf{G G}^{\dagger}+\beta \mathbf{G}_{\mathbf{b e}}^{(\mathbf{i})} \mathbf{G}_{\mathbf{b e}}^{(\mathbf{i})^{\dagger}}$ being singular, $\left(N_{A}-1\right)+\left(N_{B}-1\right) \geq N_{E}$ has to be ensured.

Second, for Eve's antenna number, $N_{E}$, it is related to an assumption on the capability of Eve. $N_{E}=N_{B}$ is typically assumed to mean that Eve has the same capability as Bob. $N_{E}$ can also be considered as the number of colluding eavesdroppers, each equipped with one antenna.

5.2. Multiple Receiving Antennas for Bob. In this paper, the transmission between Alice and Bob adopts the MISO strategy; that is, Bob only uses one antenna for information receiving. In fact, Bob can use more than one antenna to receive information signals, because he has $N_{B}$ antennas. Although authors in [13] have pointed out that the secrecy capacity, Cs, does not necessarily grow linearly with the number of receive dimensions, it still might result in a better performance than the MISO strategy. For such MIMO cases, it tends to be more difficult to analyze the statistical expectation of $\overline{C s_{2}}$. Furthermore, the antenna selection problem becomes combinatorial NP hard for Bob with multiple receiving antennas. In future work, further efforts need to be made to solve these problems. 
TABLE 1: Comparison results for Bob's antennas.

\begin{tabular}{lcccc}
\hline$i$ & 1 & 2 & 3 & 4 \\
\hline$\left\|\widetilde{h_{a b}^{(i)}}\right\|$ & 2.16 & 3.03 & 2.67 & 2.79 \\
\hline
\end{tabular}

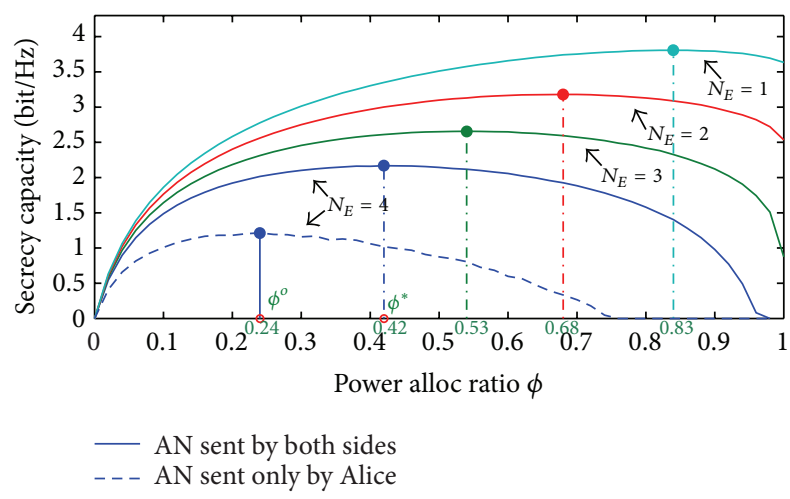

FIGURE 2: Secrecy capacity versus power allocation ratio $\phi$.

\section{Simulation Results}

In the simulation scenario (see Figure 4), topology is set as $(-2,2) \mathrm{km} \times(-2,2) \mathrm{km}$. Alice and Bob are located at $(-0.5,0)$ and $(0.5,0)$, respectively, with $N_{A}=8$ and $N_{B}=4$. Simulation parameters are set to the following values: $P_{A}=P_{B}=0.1 \mathrm{~W}$, $\lambda=10^{-12.81}$, and $\kappa=3.76$. The noise power density is $-180 \mathrm{dBm} / \mathrm{Hz}$, which gives a noise power of $\sigma_{n_{b}}^{2}=5 * 10^{-15} \mathrm{~W}$ within a bandwidth of $5 \mathrm{MHz}$. Such configurations result in an SNR of $4.77 \mathrm{~dB}$ at Bob. The channel fading between Alice and Bob is generated randomly.

Optimal design of this scenario is analyzed first. According to the antenna assignment method in Section $4.1, \mathbf{h}_{\mathbf{a b}}^{(\mathbf{i})}$ $\left(1 \leq n \leq N_{B}\right)$ is computed for each of Bob's antennas, shown in Table 1.

Bob will therefore assign the 2nd antenna as the receiving antenna, and the remaining 3 ones for $\mathrm{AN}$ emission.

Figure 2 shows the power allocation ratio $\phi$ and the corresponding secrecy capacity computed according to Section 4.2. Eve's antenna number, $N_{E}$, varies from 1 to 4 , and the optimal power allocation ratio, $\phi^{*}$, is obtained for each condition. Secrecy capacity drops drastically with the increase of $N_{E} . \phi^{*}$ also decreases so that a greater proportion of the power should be assigned to $\mathrm{AN}$ in order to resist a more powerful eavesdropper. For $N_{E}=4$, the dashed-line also shows the secrecy capacity when $\mathrm{AN}$ is sent only by Alice, and $\phi^{\circ}$ is the optimal power allocation ratio for this condition.

Figure 3 compares the secrecy capacity for $3 \mathrm{AN}$ strategies at different SNR levels. To be fair, model (b) and model (c) are configured under the same resource condition as follows: $N_{A}=6, N_{B}=4, N_{E}=2$, and $P_{B}=P_{A}$. As it can be seen from Figure 3, the strategy of model (c) has better performance. In addition, positive secrecy capacity can be achieved even when SNR is fairly low. For model (a), only Alice sends AN, therefore, $P_{B}=0$, and the secrecy capacity is still taken under the optimal power allocation ratio, denoted as $\phi^{\circ}$ in Figure 3.

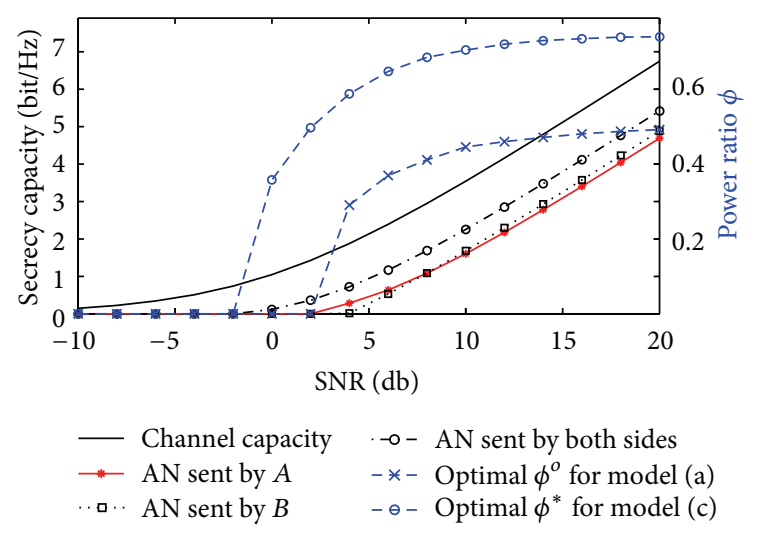

FIGURE 3: Comparison of 3 strategies in terms of secrecy capacity.

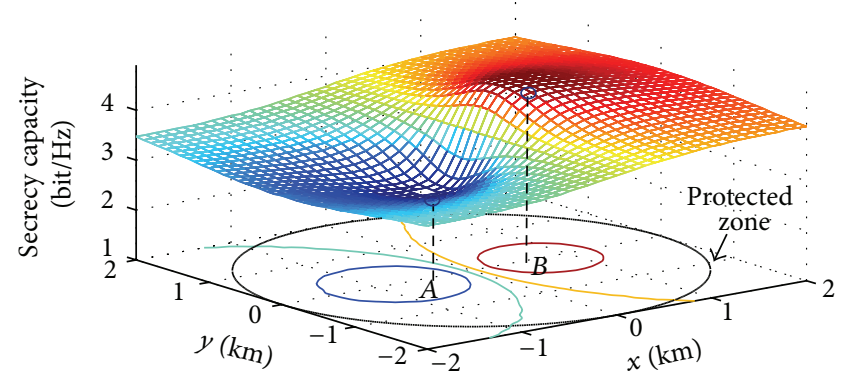

FIGURE 4: Impact of Eve's location on the secrecy capacity.

The achieved secrecy capacity of model (c) still outperforms that of model (a), but at the cost of extra AN power emitted by Bob.

In Figure 4, rather than the average secrecy capacity, we turn to see the distribution details of secrecy capacity when the attacker appears at various locations of the topology. When the eavesdropper approaches Alice, the secrecy capacity is degraded greatly due to Eve's superior channel condition compared with Bob. With the help of AN emitted by Alice, however, positive secrecy capacity is still possible. When Eve is located near Bob, high secrecy capacity is achieved, disregarding the fact that channel conditions for Eve and Bob are similar. This is a result of the strong interfering signal emitted by Bob, making it difficult for Eve to cancel the randomized $\mathrm{AN}$.

\section{Conclusion}

In this paper, artificial noise sent by both Alice and Bob was studied. We proposed an efficient scheme, called "bipolarbeamforming optimization," to optimally configure the proposed model. Secrecy capacity was analyzed based on Eve's stochastic channel condition and spatial distribution. Maximum secrecy capacity can be achieved by adopting the proposed optimization technique to allocate their antenna and power resources. Research work of this paper can be extended to a more general configuration where Bob adopts more than one antennas for receiving the information signals. 


\section{Acknowledgments}

This work was supported in part by the Natural Science Foundation of China (NSFC) under Grant no. 60932003 and no. 61271220. Thanks for the Editor SHEN Hao's dedicated work. The authors are also grateful to the anonymous reviewers for their helpful comments and constructive suggestions on the original submission manuscript. Professor LI Jianhua (email:1jh888@sjtu.edu.cn) from the Department of Electronic Engineering, Shanghai Jiao Tong University, has provided useful instructions on the revision of our paper.

\section{References}

[1] A. D. Wyner, “The wire-tap channel," The Bell System Technical Journal, vol. 54, no. 8, pp. 1355-1387, 1975.

[2] I. Csiszár and J. Körner, "Broadcast channels with confidential messages," IEEE Transactions on Information Theory, vol. 24, no. 3, pp. 339-348, 1978.

[3] Y. Liang, H. V. Poor, and S. Shamai, "Secure communication over fading channels," IEEE Transactions on Information Theory, vol. 54, no. 6, pp. 2470-2492, 2008.

[4] F. Oggier and B. Hassibi, "The secrecy capacity of the MIMO wiretap channel," IEEE Transactions on Information Theory, vol. 57, no. 8, pp. 4961-4972, 2011.

[5] H. Shen, S. Xu, X. Song, and Y. Chu, "Delay-dependent $H_{\infty}$ filtering for stochastic systems with Markovian switching and mixed mode-dependent delays," Nonlinear Analysis. Hybrid Systems, vol. 4, no. 1, pp. 122-133, 2010.

[6] S. He and F. Liu, "Adaptive observer-based fault estimation for stochastic Markovian jumping systems," Abstract and Applied Analysis, vol. 2012, Article ID 176419, 11 pages, 2012.

[7] H. Shen, S. Xu, X. Song, and J. Luo, "Delay-dependent robust stabilization for uncertain stochastic switching systems with distributed delays," Asian Journal of Control, vol. 11, no. 5, pp. 527-535, 2009.

[8] S. He and F. Liu, "Robust stabilization of stochastic Markovian jumping systems via proportional-integral control," Signal Processing, vol. 91, no. 11, pp. 2478-2486, 2011.

[9] H. Shen, S. Xu, J. Lu, and J. Zhou, "Passivity-based control for uncertain stochastic jumping systems with mode-dependent round-trip time delays," Journal of the Franklin Institute. Engineering and Applied Mathematics, vol. 349, no. 5, pp. 1665-1680, 2012.

[10] Z.-G. Wu, P. Shi, H. Su, and J. Chu, "Passivity analysis for discrete-time stochastic markovian jump neural networks with mixed time delays," IEEE Transactions on Neural Networks, vol. 22, no. 10, pp. 1566-1575, 2011.

[11] Z. Wu, H. Su, J. Chu, and W. Zhou, "Improved result on stability analysis of discrete stochastic neural networks with time delay," Physics Letters A, vol. 373, no. 17, pp. 1546-1552, 2009.

[12] R. Negi and S. Goel, "Secret communication using artificial noise," in Proceedings of the 62nd IEEE Vehicular Technology Conference (VTC '05), vol. 3, pp. 1501-1506, September 2005.

[13] S. Goel and R. Negi, "Guaranteeing secrecy using artificial noise," IEEE Transactions on Wireless Communications, vol. 7, no. 6, pp. 2180-2189, 2008.

[14] Y. Zhu, Y. Zhou, S. Patel, X. Chen, L. Pang, and Z. Xue, "Artificial noise generated in MIMO scenario: optimal power design," IEEE Signal Processing Letters, vol. 20, no. 10, pp. 964-967, 2013.
[15] X. Zhou and M. R. McKay, "Secure transmission with artificial noise over fading channels: achievable rate and optimal power allocation," IEEE Transactions on Vehicular Technology, vol. 59, no. 8, pp. 3831-3842, 2010.

[16] P. Huang and X. Wang, "Secrecy enhancement with artificial noise in decentralized wireless networks: a stochastic geometry perspective," in Proceedings of the IEEE Wireless Communications and Networking Conference, pp. 935-940, April 2013.

[17] A. Mukherjee and A. L. Swindlehurst, "Robust beamforming for security in MIMO wiretap channels with imperfect CSI," IEEE Transactions on Signal Processing, vol. 59, no. 1, pp. 351-361, 2011.

[18] W. Li, M. Ghogho, B. Chen, and C. Xiong, "Secure communication via sending artificial noise by the receiver: outage secrecy capacity/region analysis," IEEE Communications Letters, vol. 16, no. 10, pp. 1628-1631, 2012.

[19] A. K. Khandani, "Methods for spatial multiplexing of wireless two-way channels," US patent US7817641 B1, Application number US 11/581,427, 2010.

[20] M. Jain, J. I. Choi, T. Kim et al., "Practical, real-time, full duplex wireless," in Proceedings of the 17th Annual International Conference on Mobile Computing and Networking (MobiCom '11), pp. 301-312, September 2011.

[21] S. W. Kim, Y. J. Chun, and S. Kim, "Co-channel interference cancellation using single radio frequency and baseband chain," IEEE Transactions on Communications, vol. 58, no. 7, pp. 21692175,2010

[22] H. Gao, P. J. Smith, and M. V. Clark, “Theoretical reliability of MMSE linear diversity combining in rayleigh-fading additive interference channels," IEEE Transactions on Communications, vol. 46, no. 5, pp. 666-672, 1998.

[23] M. Ghogho and A. Swami, "Physical-layer secrecy of MIMO communications in the presence of a poisson random field of eavesdroppers," in Proceedings of the IEEE International Conference on Communications Workshops (ICC '11), pp. 1-5, June 2011. 


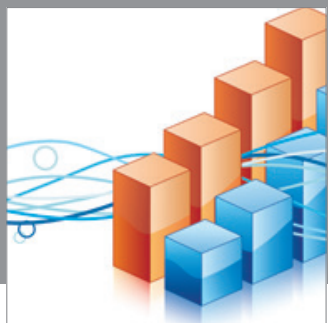

Advances in

Operations Research

mansans

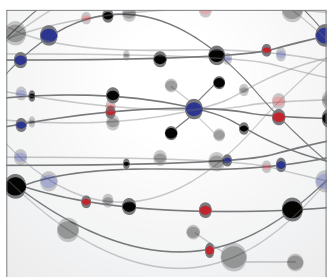

The Scientific World Journal
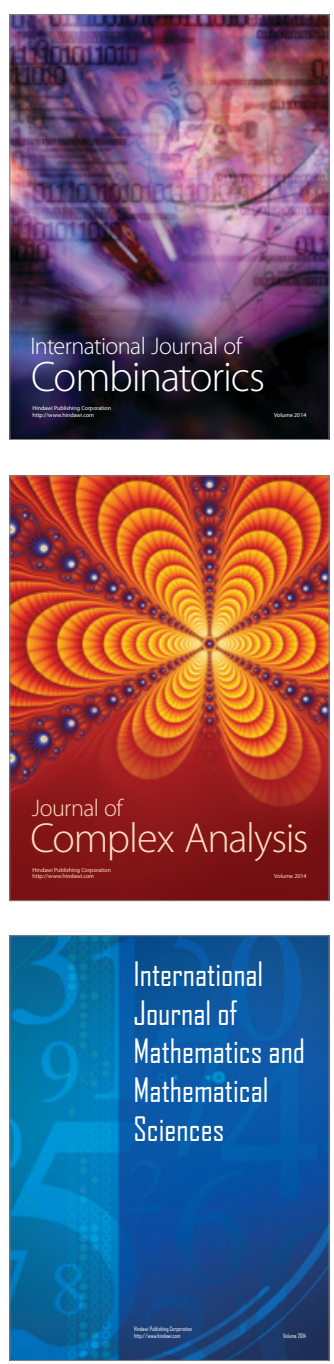
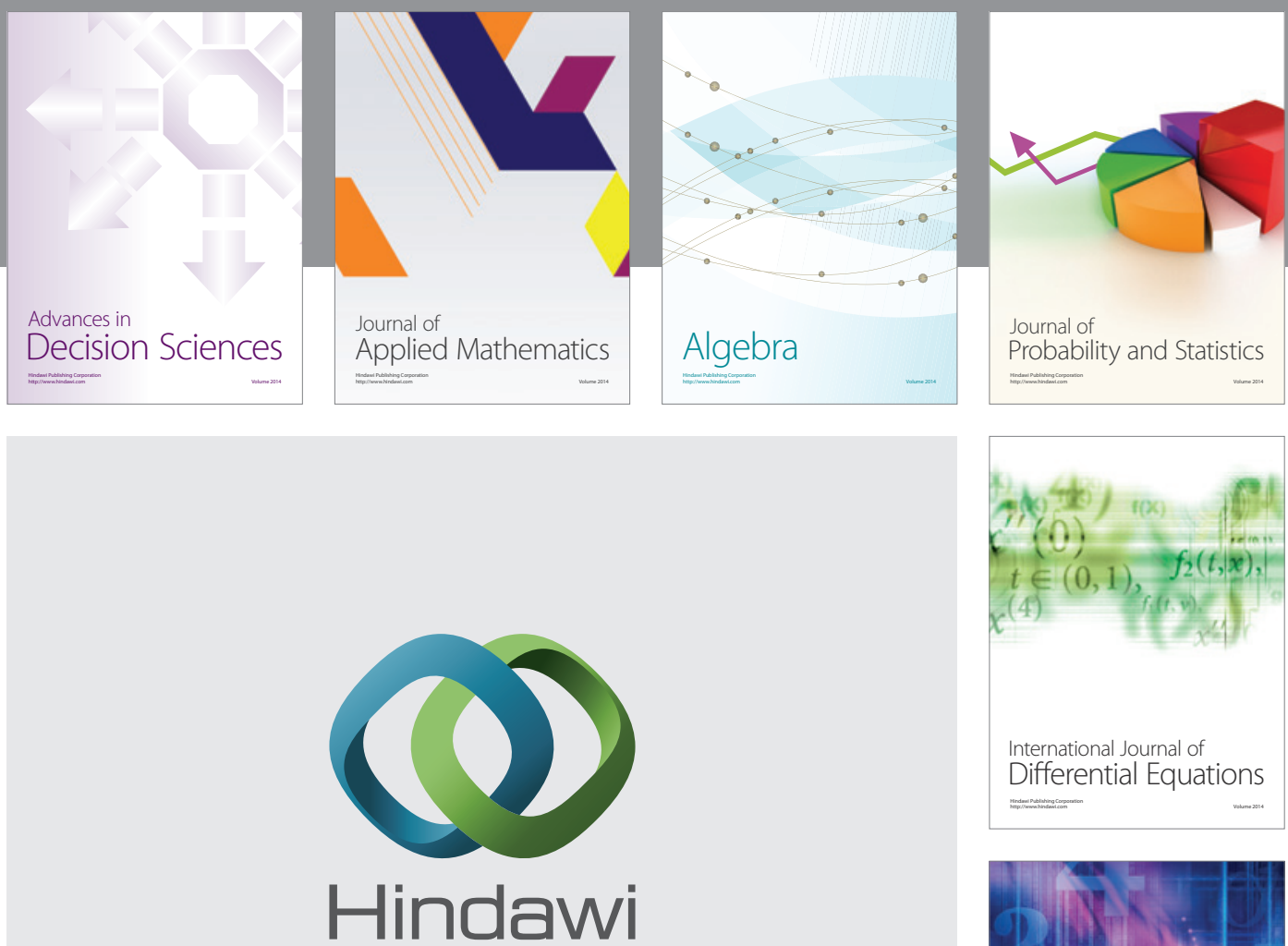

Submit your manuscripts at http://www.hindawi.com
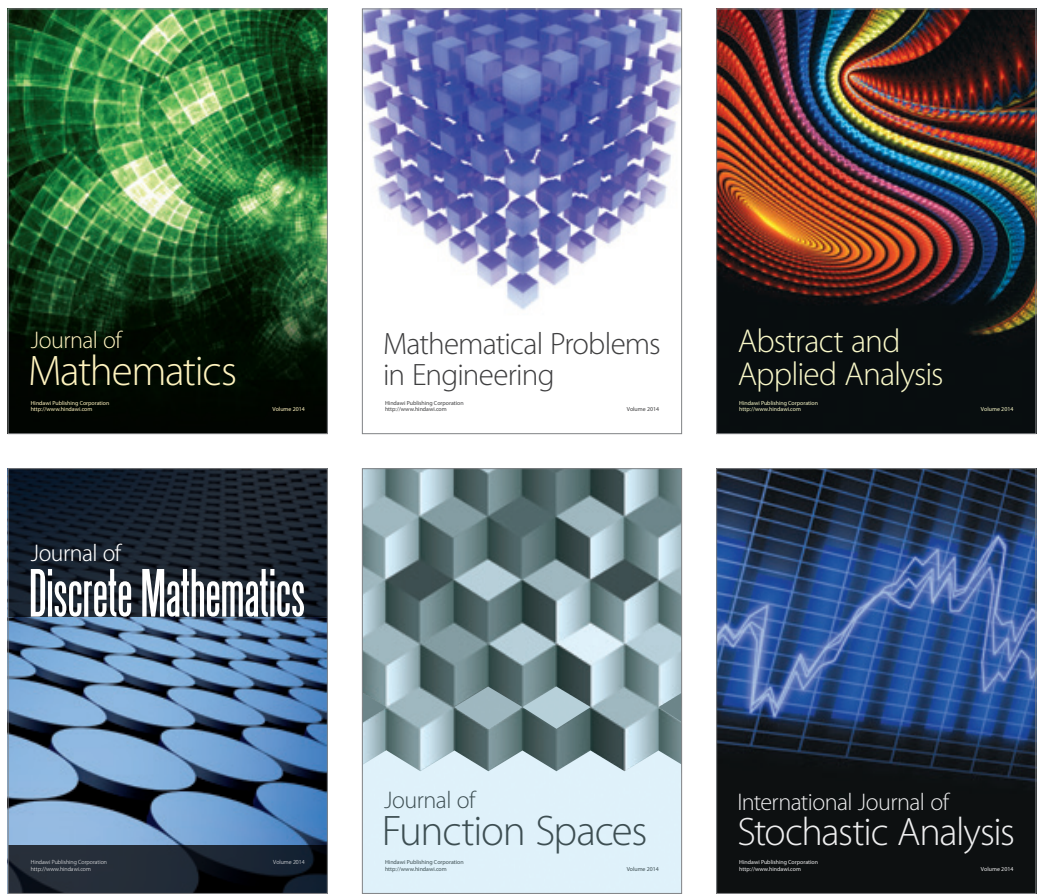

Journal of

Function Spaces

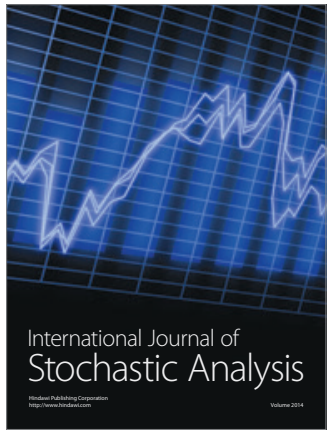

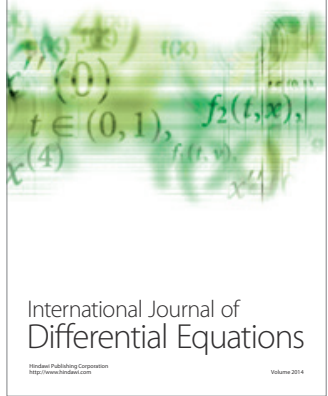
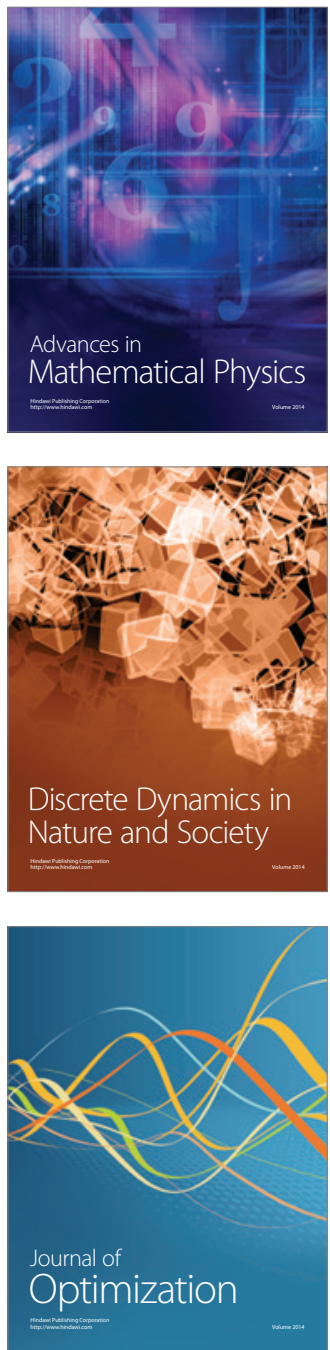